Proc. Estonian Acad. Sci. Eng., 2004, 10, 3, 157-172

\title{
Comparison of photon recycling effect in GaAs and GaN structures
}

\begin{abstract}
Enn Velmre and Andres Udal
Department of Electronics, Tallinn University of Technology, Ehitajate tee 5, 19086 Tallinn, Estonia; evelmre@ttu.ee; audal@va.ttu.ee

Received 6 April 2004

Abstract. Extending earlier studies of photon recycling effect in GaAs, estimations are found for a new promising wide band-gap semiconductor GaN. Relying on available data, approximately one order higher radiative recombination coefficient and absorption coefficients than in GaAs and equal or greater Urbach energies than in GaAs are valid for $\mathrm{GaN}$. The distance-dependent radiative recombination transfer functions are introduced and studied for typical charge carrier distribution cases. In spite of high absorption rates of $\mathrm{GaN}$, the estimations show approximately one order of magnitude higher photon recycling efficiency than in the case of GaAs. By numerical simulations, the possibility of appearance of the $\mathrm{S}$-shape forward $I / V$ characteristics of $p-i-n$ structures due to strong photon recycling is shown.
\end{abstract}

Key words: photon recycling, recombination radiation reabsorption, Gallium arsenide, Gallium aitride, radiative recombination transfer function, one-dimensional drift-diffusion simulations, $p-i-n$ structures.

\section{INTRODUCTION}

The recombination radiation reabsorption phenomenon in direct band-gap semiconductors was first described by Dumke $\left[{ }^{1}\right]$. This so-called photon recycling $\left[^{2}\right]$ effect has at least two consequences. First of all, it is efficiently compensating the channel of radiative recombination of charge carriers and, secondly, it is causing an additional electron-hole pair diffusion, if there is a gradient of radiative recombination distribution or, in other words, a gradient of electron or hole concentrations. Dumke found that the photon recycling gives rise to a carrier diffusion described by an effective diffusion coefficient, called the photon diffusion coefficient: 


$$
D_{\mathrm{ph}}=\frac{\left\langle\alpha^{-2}\right\rangle}{3 \tau_{\mathrm{r}}},
$$

where $\left\langle\alpha^{-2}\right\rangle$ is a suitable average over the inverse squared absorption coefficient and $\tau_{\mathrm{r}}$ is the radiative lifetime.

Dumke estimated the magnitude of the photon diffusion coefficient in the order of $1 \mathrm{~cm}^{2} / \mathrm{s}$, which is quite small compared to actual charge carrier diffusivities. So it was concluded that the photon recycling should have only a minor effect on the carrier transport in semiconductors with high absorption coefficient at photon energies close to the band edge.

Since 1960s, GaAs has been one of the most frequently used optoelectronic materials. In 1970, Alferov with his collaborators $\left[^{3}\right]$ accomplished the continuously operating $\mathrm{GaAs} / \mathrm{Al}_{\mathrm{x}} \mathrm{Ga}_{1-\mathrm{x}} \mathrm{As}$ heterostructure laser. This development gave a new impact on GaAs based materials and device research.

In the second half of $1970 \mathrm{~s}$, the power diode $\left[{ }^{4}\right]$ and thyristor $\left[{ }^{5}\right]$ structures, based on a lightly doped $n$-type epitaxial GaAs layer, were manufactured in Alferov's laboratory. Subsequent experimental $\left[{ }^{6,7}\right]$ and theoretical $\left[{ }^{8,9}\right]$ investigations of these structures revealed a remarkable influence of photon recycling on the electrical characteristics of the devices. It was shown that besides intrinsic photon recycling in the $n$-base region, a relatively long wave recombination radiation, emitted from the $p^{+}$-emitter and absorbed in the far edge of the $n$-base, has strong influence on the $I / V$ characteristics of the device at high current densities.

Expectations were high, but nowadays GaAs-based power devices are not especially widespread. Nevertheless, investigation of photon recycling effect is still topical, but now, for example, in connection with solar cell design (AlGaAs/GaAs/AlGaAs heterostructure $\left[{ }^{10}\right]$ ) or photoluminescence spectra analysis $\left(n-\operatorname{InP}\left[{ }^{11}\right]\right.$ and $\left.\operatorname{InP} / \operatorname{InPGaAs}\left[{ }^{12}\right]\right)$.

At present, some wide band-gap semiconductors as certain SiC polytypes, $\mathrm{GaN}$ and even $\mathrm{C}$ (diamond) are considered as promising future materials. Apart from others, gallium nitride has two direct band-gap type polytypes, $2 \mathrm{H}-\mathrm{GaN}$ and $3 \mathrm{C}-\mathrm{GaN}$, and therefore the photon recycling would play an important role in structures based on these materials. But, as usual in case of novel materials, some values of $\mathrm{GaN}$ electrical and optical parameters are still uncertain and causing problems in quantitative analysis of the photon recycling effect. The situation is quite different from GaAs, for which a reliable set of all necessary parameters is available.

In this paper, we will discuss a comparison methodology for the photon recycling effect in $\mathrm{GaAs}$ and $2 \mathrm{H}-\mathrm{GaN}$. In quantitative estimations for $\mathrm{GaN}$ we will choose from rather uncertain published data those that propose approximately one order higher radiative recombination coefficient $B$ and absorption coefficient $\alpha$ than in GaAs, and equal or greater Urbach energy $E_{\mathrm{Urb}}$ than in GaAs. From semiconductor device simulation viewpoint, the photon recycling effect is associated with modelling of radiative recombination $R_{\mathrm{r}}$ and radiative generation terms $G_{\mathrm{r}}$ in 
continuity equations. We shall discuss also one-dimensional numerical simulation results for $p-i-n$ structures where strongly non-uniform charge carrier density distributions are remarkably affected by the recombination radiation.

\section{MODELLING OF THE GENERATION TERM}

Probably the first completely numerical simulator, accounting for recombination radiation reabsorption effect in multijunction direct band-gap semiconductor structures, was developed by Velmre with collaborators $\left[{ }^{13,14}\right]$. The mathematical model used in this one-dimensional (1D) simulator was based on the finite difference solution of the full set of semiconductor equations in drift-diffusion approximation with boundary conditions on the terminal contacts of the structure. The electron and hole continuity equations have been modified to take account of the carrier generation due to reabsorption of the band-to-band recombination radiation. Simulation results $\left[{ }^{13,14}\right]$ of forward steady-state characteristics of GaAs $p-i-n$ diodes demonstrated a significant role of the photon recycling on the behaviour of the power devices, especially at a high operation current level. Unfortunately, the Seidman-Choo iterative scheme $\left[{ }^{15}\right]$, which was implemented in our early simulators, was not efficient at high forward currents. Later on, a more efficient coupled solution numerical approach $\left[{ }^{16}\right]$ was developed that removed most of the convergence problems at high injection and high radiative recombination-generation levels.

Assuming band-to-band radiation recombination and neglecting traps, the electron and hole recombination-generation rates $R_{\mathrm{n}}$ and $R_{\mathrm{p}}$ in the electron and hole continuity equation are equal and given by

$$
R_{\mathrm{n}}=R_{\mathrm{p}}=R_{\mathrm{nr}}+R_{\mathrm{r}}-G_{\mathrm{pr}},
$$

where the non-radiative recombination rate $R_{\mathrm{nr}}$ is a sum of Shockley-Read-Hall and Auger recombination rates, $G_{\mathrm{pr}}$ denotes generation of electron-hole pairs due to the readsorbed photons and the radiative recombination rate $R_{\mathrm{r}}$ is written as

$$
R_{\mathrm{r}}=B\left(n p-n_{i}^{2}\right)
$$

where $B$ is the band-to-band radiation recombination coefficient. For GaAs the value of $B$ is known rather well even for different doping levels and temperatures. We have used here $B=7.21 \times 10^{-10} \mathrm{~cm}^{3} / \mathrm{s}\left[{ }^{17}\right]$, which is a standard value for low-doped GaAs at $300 \mathrm{~K}$. Unfortunately, reference sources suggest for $\mathrm{GaN}$ still very diverse values of the coefficient $B$ within a very broad range: e.g., from $2.4 \times 10^{-11}\left[{ }^{18}\right]$ and $5 \times 10^{-11}\left[{ }^{19}\right]$ to $1.1 \times 10^{-8} \mathrm{~cm}^{3} / \mathrm{s}\left[{ }^{20}\right]$. However, considering that widely used Roosbroeck and Shockley theory $\left[{ }^{21}\right]$, based on an application of the principle of detailed balance, associates higher absorption coefficient $\alpha$ with higher radiation efficiency (see below) and several experiments have shown high $\alpha$ values at photon energies $E$ close to bandgap $E_{\mathrm{g}}$ for $2 \mathrm{H}-\mathrm{GaN}$, 
e.g., $2.6 \times 10^{4}\left[{ }^{18}\right], 6.3 \times 10^{4}\left[{ }^{22}\right]$ and $8.8 \times 10^{4} \mathrm{~cm}^{-1}\left[{ }^{20}\right]$ (over typical value of GaAs $\left.0.78 \times 10^{4} \mathrm{~cm}^{-1}\left[{ }^{23,24}\right]\right)$, in the present study we have relied on the paper by Muth et al. $\left[{ }^{20}\right]$ which gives coefficient $B$ about 15 times and the shortwave absorption coefficient $\alpha$ about 12 times as high as in GaAs.

The 1D treatment of photon recycling processes must operate with photon emission plane coordinate $x^{\prime}$ and photon absorption plane coordinate $x$ and with the distance between these planes $u \equiv\left|x-x^{\prime}\right|$, although propagation and absorption of photons are in reality of 3D nature. Several authors have discussed that problem $\left[{ }^{25-27}\right]$. Our earlier publications $\left.{ }^{13,14}\right]$ have followed mainly the approach of Kuriyama et al. $\left.{ }^{26}\right]$. If monochromatic photon flux, emitted at $x^{\prime}$, would be parallel to the $x$ axis and equally distributed between two possible directions, the probability that a photon reaches coordinate $x$ would be $P=\exp (-\alpha u) / 2$ and the probability that a photon is absorbed at $x$ in a layer $\mathrm{d} x$ becomes

$$
-\frac{\partial P}{\partial u} \mathrm{~d} x=\frac{1}{2} \alpha \exp (-\alpha u) \mathrm{d} x
$$

If a realistic 3D picture of uniformly distributed photon propagation directions is calculated and integration over all possible angles $\theta$ is performed, the last expression is replaced by

$$
-\frac{\partial P}{\partial u} \mathrm{~d} x=-\frac{1}{2} \alpha \mathrm{d} x \int_{0}^{\pi / 2} \exp (-\alpha u / \cos \theta) \frac{\sin \theta}{\cos \theta} \mathrm{d} \theta=-\frac{1}{2} \alpha \operatorname{Ei}(-\alpha u) \mathrm{d} x,
$$

where $\operatorname{Ei}(z) \equiv \int_{-\infty}^{z} \exp (t) / t \mathrm{~d} t$ with $z<0$ denotes the exponential integral function. This formulation may be used for homogeneous $\alpha$ within the limits of the described area $\left(x \ldots x^{\prime}\right)$.

Introducing the normalized emission spectrum $\rho(E)$ and the coefficient of internal photoeffect $\beta(E)$ (i.e., probability that an absorbed photon with energy $E$ creates an electron-hole pair), integration over photon energies $E$ and photon emission (recombination) coordinates $x^{\prime}$ yields the following general expression for the photon recycling generation rate $G_{\mathrm{pr}}$ at an arbitrary point on the $x$ axis in case of homogeneously doped layer with thickness $\left.w{ }^{14,24}\right]$ :

$$
G_{\mathrm{pr}}(x)=\int_{0}^{w} R_{\mathrm{r}}\left(x^{\prime}\right) F(u) \mathrm{d} u
$$

where

$$
F(u)=-\frac{1}{2} \int_{0}^{\infty} \beta(E) \rho(E) \alpha(E) \operatorname{Ei}(-\alpha(E) u) \mathrm{d} E .
$$

The following remarks should be made in respect of the model defined by Eqs. (5) and (6). Although in Eq. (6) the function $\operatorname{Ei}(-\alpha u)$ and thereby also 
$F(u)$ exhibit $\ln |u|$-type infinity at $u \rightarrow 0$, the integration in Eq. (5) gives still finite generation rates if the limit $u \rightarrow 0$ is correctly handled. If $\beta=1$ and the normalized radiation spectrum $\int_{0}^{\infty} \rho(E) \mathrm{d} E=1$ is used, then integral over all distances $\int_{0}^{\infty} F(u) \mathrm{d} u$ approaches the value $1 / 2$. In practical calculations the necessary range of photon energies may be usually restricted by $0.15 \mathrm{eV}$ around the peak energy of radiation spectrum. If necessary, the radiative recombination transfer function $F(u)$ may be calculated for any interval of photon energies, e.g., separately for the long-wave and short-wave part of the spectrum.

Throughout the present study, internal and external interface reflection effects have been neglected. This simplification is justified for sufficiently thick homojunction power device structures where the main part of the base region is far from internal ( $p n$-junction) or external (contacts, structure surface) reflective interfaces. Also in the calculations below we have assumed the lossless generation of new electron-hole pairs by reabsorbed photons $(\beta=1)$.

In principle, both measured and calculated spectra may be used in numerical simulations. In this paper, the van Roosbroeck-Shockley relationship $\left[{ }^{21}\right]$

$$
\rho(E)=c_{\mathrm{n}} \bar{n}^{2}(E) \alpha(E) E^{2}\left[\exp \left(E / k_{\mathrm{B}} T\right)-1\right]^{-1}
$$

was applied for the calculation of semitheoretical emission spectrum by empirical absorption data $\alpha(E)$ and refractive index $\bar{n}(E)$. The coefficient $c_{\mathrm{n}}$ was defined from the normalization condition $\int_{0}^{\infty} \rho(E) \mathrm{d} E=1$.

Experience shows that for temperatures equal and above the room temperature, when exciton peaks and impurity-related absorption effects become less noticeable, the energy dependence of the absorption coefficient near the band-gap energy may be approximated as follows:

$$
\alpha(E)=\left\{\begin{array}{cc}
\alpha_{\mathrm{m}} \exp \left(\left(E-E_{\mathrm{m}}\right) / E_{\mathrm{Urb}}\right), & E \leq E_{\mathrm{m}} \\
\alpha_{\mathrm{m}}+A_{\mathrm{h}}\left(E-E_{\mathrm{m}}\right), & E \geq E_{\mathrm{m}}
\end{array}\right.
$$

where energy $E_{\mathrm{m}}$ defines maximum of the radiation spectrum in Eq. (7) and is close to the material band-gap $E_{\mathrm{m}} \approx E_{\mathrm{g}}, \alpha_{\mathrm{m}}$ is the characteristic absorption coefficient at $E=E_{\mathrm{m}}, A_{\mathrm{h}}$ characterizes the increase of $\alpha$ at higher photon energies (less important here due to narrow $E$ range) and $E_{\mathrm{Urb}}$ is the wellknown "Urbach tail" energy of $10 \mathrm{meV}$ range $\left[{ }^{28}\right]$ that characterizes exponential decrease of the absorption coefficient at lower photon energies (and consequently propagation of longwave recombination radiation). For GaAs we have used the following values of the parameters: $E_{\mathrm{m}}=1.424 \mathrm{eV}, \alpha_{\mathrm{m}}=7.8 \times 10^{3} \mathrm{~cm}^{-1}$, $E_{\mathrm{Urb}}=6.58 \mathrm{meV}$ and $A_{\mathrm{h}}=3 \times 10^{4} \mathrm{~cm}^{-1} / \mathrm{eV}\left[{ }^{24}\right]$ that give an approximation error less than $\pm 5 \%$ for the experimental low-doped $n$-GaAs data $\left[{ }^{23}\right]$ at $T=300 \mathrm{~K}$ in the photon energy range from 1.39 to $1.59 \mathrm{eV}$. In the present work we have used energy-independent $\bar{n}=3.64$ for GaAs $\left[{ }^{29}\right]$. In the case of rather poorly characterized $2 \mathrm{H}-\mathrm{GaN}$ we have taken $E_{\mathrm{m}}=3.42 \mathrm{eV}, \alpha_{\mathrm{m}}=8.8 \times 10^{4} \mathrm{~cm}^{-1}$, $E_{\mathrm{Urb}}=13 \mathrm{meV}$ and $\left.A_{\mathrm{h}}=9 \times 10^{4} \mathrm{~cm}^{-1} / \mathrm{eV}^{20}\right]$ and slightly energy-dependent 


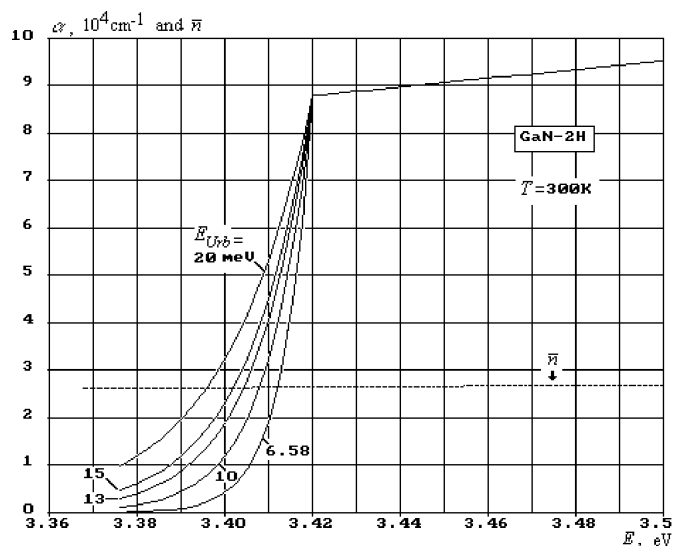

(a)

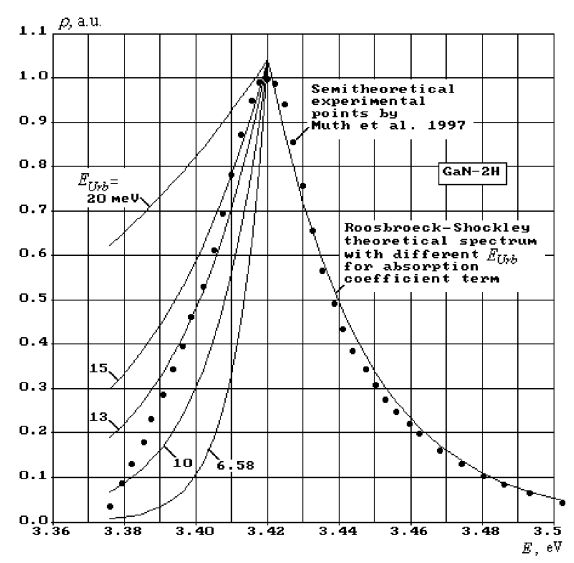

(b)

Fig. 1. Consistent specification of the absorption coefficient $\alpha(E)$ and refractive index $\bar{n}(E)$ (a) and the Roosbroeck-Shockley type recombination radiation spectrum $\rho(E)$ for $\mathrm{GaN}-2 \mathrm{H}(\mathrm{b})$. Refraction index has been taken from Ejder $\left[{ }^{30}\right]$. The varied parameter is the Urbach energy $E_{\mathrm{Urb}}$ that defines the decrease of both $\alpha(E)$ and $\rho(E)$ at lower photon energies according to Eqs. (7) and (8).

refractive index $\bar{n}(E)=2.65+C_{\mathrm{n} E}\left(E-E_{\mathrm{m}}\right) ; \quad C_{\mathrm{n} E}=0.6 / \mathrm{eV} \quad\left[{ }^{30}\right]$. Since for photon recycling very important is contribution of long-wave photons, we have below in several cases tried different $E_{\mathrm{Urb}}$ values for GaN.

Figure 1 illustrates the procedure of consistent specifying of $\alpha(E)$ and $\rho(E)$ in the case of $2 \mathrm{H}-\mathrm{GaN}$ relying on Muth et al. $\left.{ }^{20}\right]$. The Urbach energy $E_{\mathrm{Urb}}=13 \mathrm{meV}$ is selected as a basic value for the following GaN-2H calculations. It is important to notice that within the present procedure, if we use Roosbroeck-Shockley radiation spectrum (7), the Urbach energies must remain below $k_{\mathrm{B}} T$ in order to assure realistic long-wave spectrum.

\section{RESULTS}

\subsection{Investigation of the radiative recombination transfer function}

In our earlier studies, numerical simulation of different GaAs device structures $\left.{ }^{[13,14}\right]$ or analytical approximations for carrier modified diffusion coefficients and transfer factors through the device layers were considered $\left[{ }^{24}\right]$. The pure numerical simulations usually do not reveal explicitly the important interrelations between physical quantities in a wider scale. On the other hand, the simplified analytical approximations underestimate different mechanisms, in the present case first of all the long-distance influences of low-energy photons. A more systematic investigation of photon recycling effect for different distances, photon energy ranges and material parameters may be performed on the basis of the radiative recombination transfer function $F(u)$ defined by Eq. (6). 


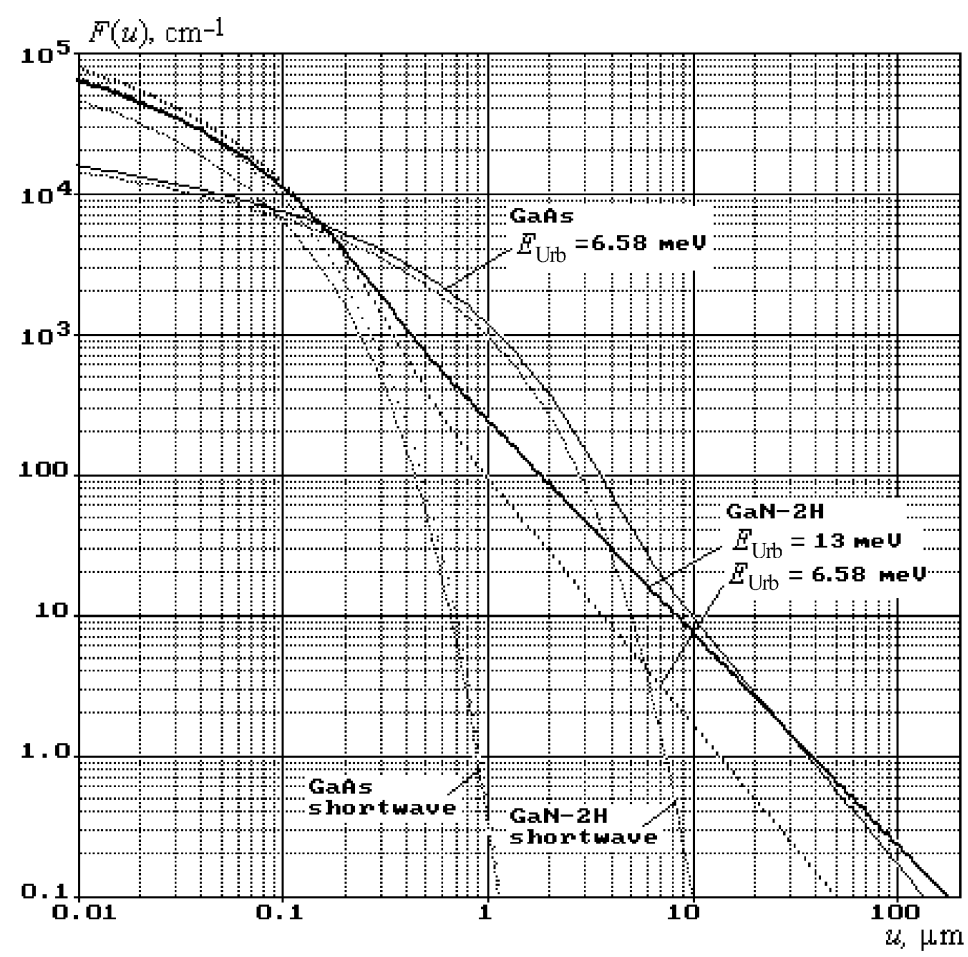

Fig. 2. Comparison of radiative recombination transfer functions (see Eqs. (5) and (6)) for GaAs and $2 \mathrm{H}-\mathrm{GaN}$.

Figure 2 compares the calculated values of $F(u)$ for GaAs and 2H-GaN. For short distances the limit approximation $\operatorname{Ei}(z \rightarrow 0) \rightarrow \ln |z|$ yields estimation $F(u) \rightarrow-\left(\alpha_{\mathrm{m}} / 2\right) \ln \left|\alpha_{\mathrm{m}} u\right|$ that is confirmed by the results in Fig. 2. This limit approximation predicts that at very small distances the ratio of $F(u)$ for two materials must approach the value $\alpha_{\mathrm{mGaN}} / \alpha_{\mathrm{mGaAs}} \approx 11$. Physically it means that at short distances remarkably below characteristic absorption length $L_{\text {char }}=1 / \alpha_{\mathrm{m}}$ (about $1.2 \mu \mathrm{m}$ for GaAs and $0.11 \mu \mathrm{m}$ for $2 \mathrm{H}-\mathrm{GaN}$ ), where the decrease of photon flux is not yet significant, the radiative generation is roughly proportional to $\alpha$ and $\mathrm{GaN}$ is approximately one order of magnitude more efficient than GaAs. At distances above about $5 L_{\text {char }}$, the short-wave $F(u)$ components in Fig. 2 exhibit abrupt decrease and the contribution of long-wave photons becomes dominant. As expected, at that the Urbach energy has a remarkable influence so that approximately two times greater $E_{\mathrm{Urb}}$ of $2 \mathrm{H}-\mathrm{GaN}$ makes it comparable with GaAs also for longer distances.

However, it should be emphasized that $F(u)$ does not take into account the difference of radiative recombination coefficients $B$ of materials. If remarkably higher $B$ of $2 \mathrm{H}-\mathrm{GaN}$ would be considered, the much more stronger phonon recycling effect of that material in comparison to GaAs would become clearly visible for all distances. Additionally, in structures the situation with spatially 
uniform charge carrier densities and respectively uniform radiative recombination rates is not interesting when considering the role of the phonon recycling effect. The strongest influence of that effect should appear in the areas of the structure with low electron and hole concentrations, where reabsorption of radiation from areas with high $\mathrm{e}-\mathrm{h}$ concentrations should remarkably change the carrier distributions. Respectively, a more advanced investigation must besides $F(u)$ take into account also spatially nonuniform $\mathrm{e}-\mathrm{h}$ distributions. Those spatially nonuniform e-h distributions may be often described by $\exp ( \pm x / L)$-type functions ( $L$ is the ambipolar diffusion length) if we consider ambipolar carrier injection (diffusion) into low doped layer or minority carrier diffusion into highdoped layer. In the case of a forward-biased $p-i-n$ structure, the injection of charge carriers into low-doped $i$-layer from both emitters forms a $\cosh (x / L)$-type carrier profile as shown in Fig. 3.

The modified photon recycling efficiency function that takes into account the difference in radiative recombination coefficients of different materials and uses typical free carrier $\cosh (x / L)$-type distribution profile (and consequently $\cosh ^{2}(x / L)$-profile for $R_{\mathrm{r}}$ ) in low-doped $i$-layer of a $p-i-n$ structure under high injection conditions, where electron and hole concentrations exceed clearly the donor and acceptor dopant concentrations, $p \approx n>>N_{D}, N_{A}$ (see Fig. 3), may be presented in the following form:

$$
F_{\mathrm{MAT}}^{\mathrm{pin}}(u)=\frac{B}{B_{\mathrm{GaAs}}} \cosh ^{2}\left(u / L_{\mathrm{a}}\right) F(u) .
$$

Equation (9) characterizes the additional generation rate near the carrier distribution minimum in low-doped region. Here $u$ varies from zero to halfwidth of the low-doped layer. An additional device parameter introduced into this treatment is the carrier ambipolar diffusion length $L_{\mathrm{a}}$. For a first approximation

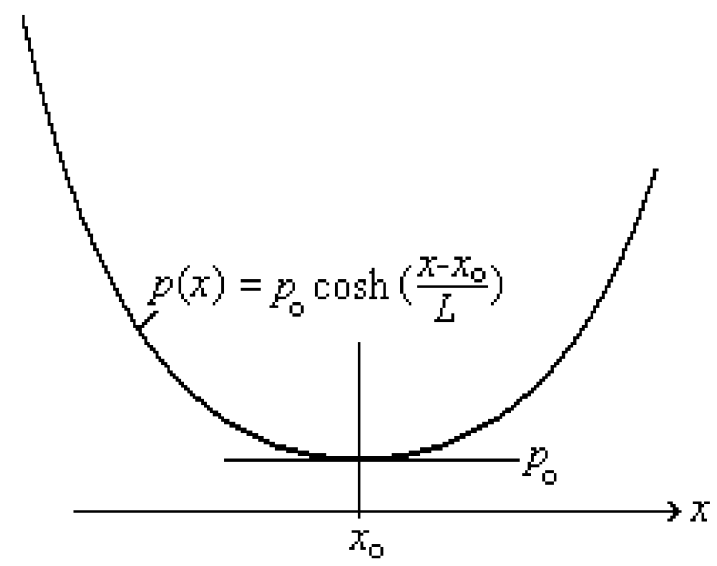

Fig. 3. The typical charge carriers $(p \approx n)$ distribution in low-doped semiconductor layers in the case of two-sided injection ( $p-i-n$ diode at high forward current density). 
this parameter may be evaluated using non-radiative carrier lifetime: $L_{\mathrm{a}}=\left(D_{\mathrm{a}} \tau_{\mathrm{nr}}\right)^{1 / 2}, D_{\mathrm{a}}=2 D_{\mathrm{n}} D_{\mathrm{p}} /\left(D_{\mathrm{n}}+D_{\mathrm{p}}\right)$; here $D_{\mathrm{n}}$ and $D_{\mathrm{p}}$ are the electron and hole diffusion coefficients. Shorter $L_{\mathrm{a}}$ gives more steep carrier gradients and respectively stronger influence of photon recycling effect near the carrier distribution minimum in a low-doped layer. Results for the modified photon recycling efficiency function, defined by Eq. (9), are presented in Fig. 4.

Figure 4 reveals clearly the remarkably stronger influence of photon recycling effect in $2 \mathrm{H}-\mathrm{GaN}$ than in GaAs. Additionally it gives the possibility to estimate the device dimensions and carrier diffusion lengths for which the photon recycling becomes important. In contrast to $F(u)$, shown in Fig. 2, which demonstrates only decreasing influence of radiative recombination at longer distances, the modified function in Fig. 4 shows clearly that starting from device dimensions of about $10 \mu \mathrm{m}$, very strong influence of photon recycling is to be expected.

As already mentioned, often over-equilibrium carrier density distributions in semiconductor device layers may be described by $\exp (u / L)$-type functions. Then in the case of low-doped layers the electron and hole concentrations are nearly equal and radiative recombination follows the $R_{\mathrm{r}} \sim p n \sim \exp ^{2}(u / L)$ shape and parameter $L$ must be treated as ambipolar diffusion length. In the case of high-

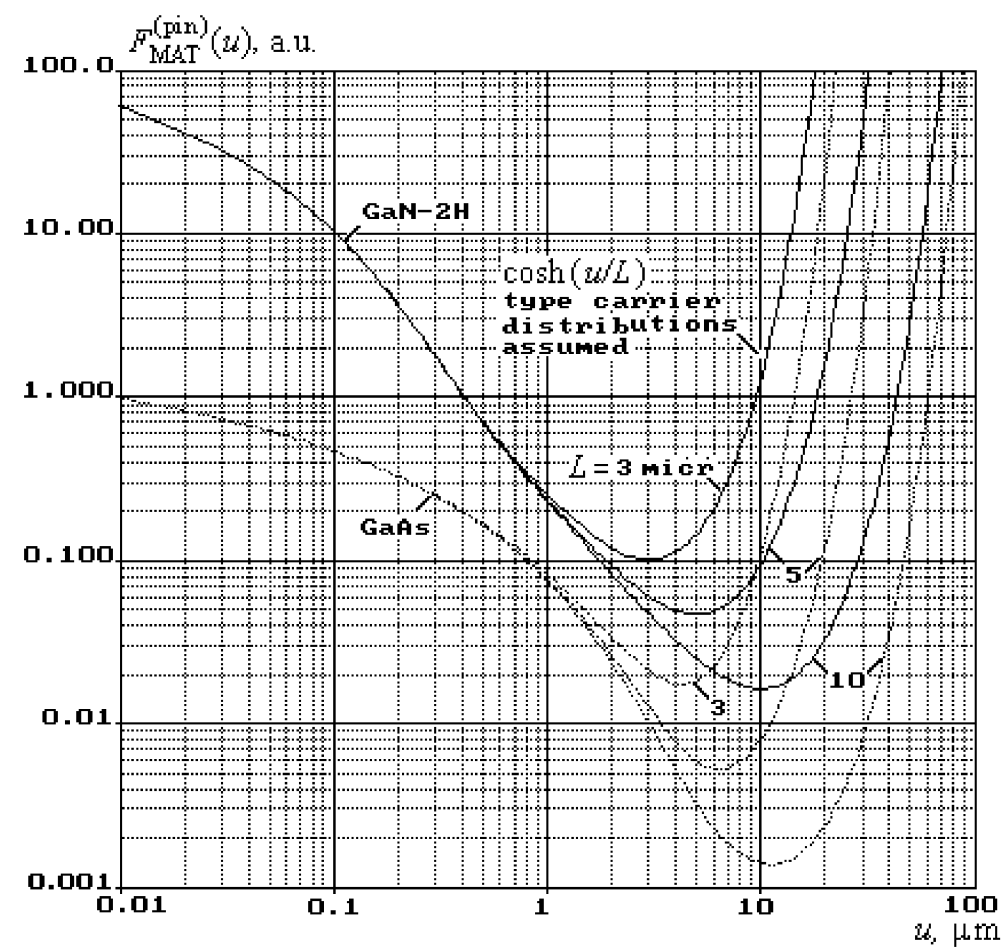

Fig. 4. The modified photon recycling efficiency function (Eq. (9)) $F_{\mathrm{MAT}}^{\mathrm{pin}}(u)$, characterizing radiative generation intensity near the carrier distribution minimum in low-doped layer of a $p-i-n$ structure. 


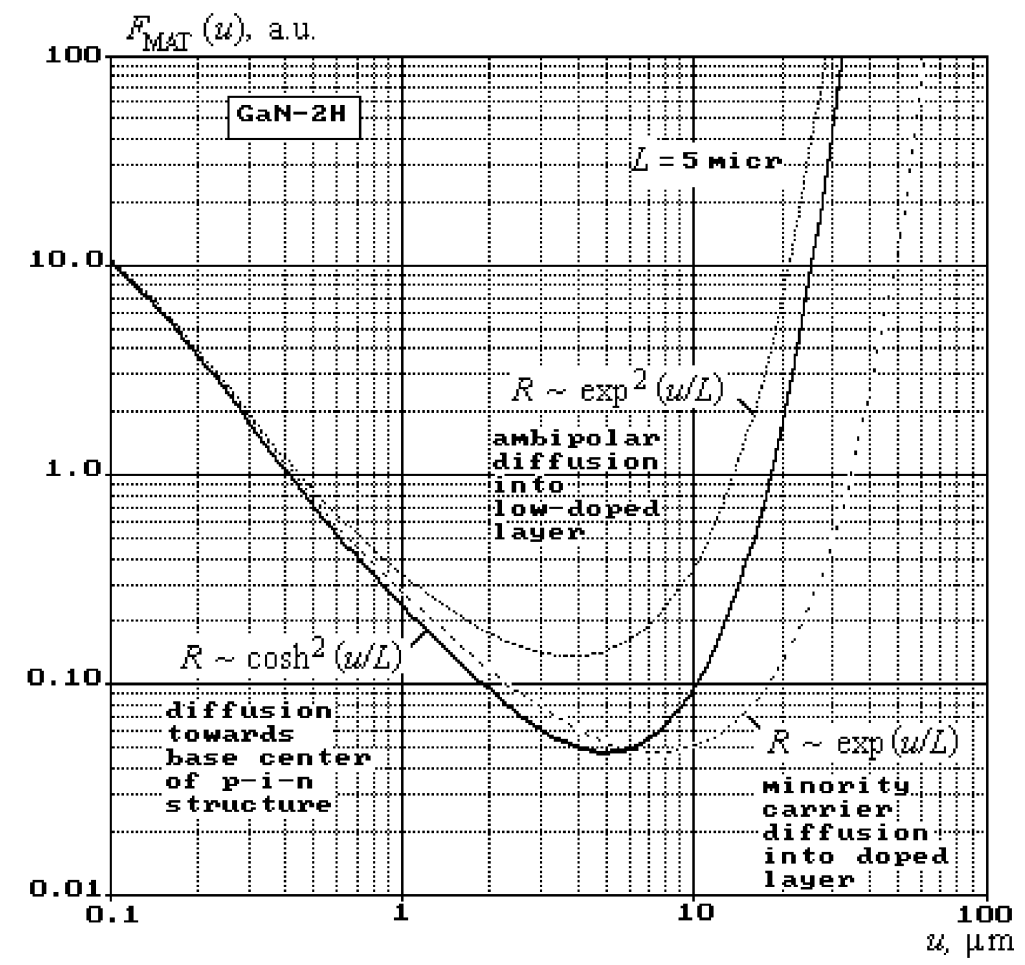

Fig. 5. Comparison of photon recycling efficiency functions for different versions of Eq. (9).

doped layers like, e.g., emitter regions of $p-i-n$ structures, where due to high majority carrier concentration the radiative recombination rate may be locally very high, only the minority carrier distribution follows $\exp (u / L)$-type function and respectively $R_{\mathrm{r}} \sim \exp (u / L)$; here $L$ is minority carrier diffusion length. The corresponding results for different versions of the modified photon recycling efficiency function (9), where instead of $\cosh ^{2}(u / L)$ either $\exp ^{2}(u / L)$ or $\exp (u / L)$ is used, are presented in Fig. 5. As one can see, the $\exp ^{2}(u / L)$-type recombination term predicts stronger influence of recombination radiation than the $\cosh ^{2}(u / L)$ type recombination term.

\subsection{Comparison of the influence of photons with different energies}

Figures 2, 4 and 5 take into account more or less wide spectrum of photon energies. However, it may be estimated that at short distances the main contribution to $F(u)$ is given by high-energy photons and at longer distances by the photons with lower energies. To investigate this question more precisely, for every distance $u$ the most efficient photon energy and energy limits towards higher and lower energies that give $90 \%$ and $99 \%$ of $F(u)$, were defined. The results are presented in Fig. 6. 


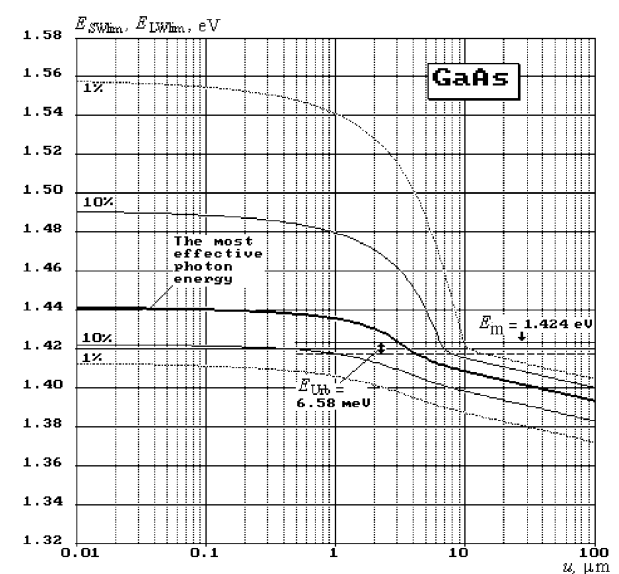

(a)

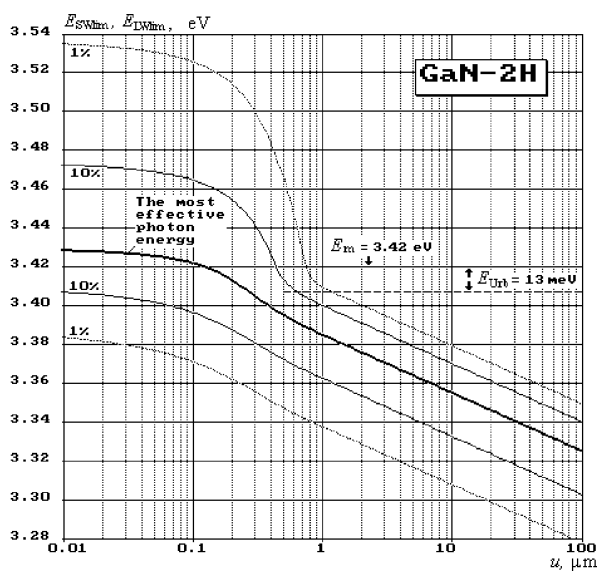

(b)

Fig. 6. Contribution of photons with different energies to the recombination radiation reabsorption in $\mathrm{GaAs}$ and $2 \mathrm{H}-\mathrm{GaN}$.

Figure 6 demonstrates clearly how the domination of short-wave photons at short distances is replaced by domination of long-wave (low-energy) photons at longer distances. The characteristic distance at which the short-wave influence is replaced by the long-wave one, is approximately from 7 to 8 times the characteristic absorption length $1 / \alpha_{\mathrm{m}}$ (that is about $1.2 \mu \mathrm{m}$ for GaAs and $0.11 \mu \mathrm{m}$ for $2 \mathrm{H}-\mathrm{GaN})$. To perform calculations with $1 \%$ accuracy in the case of GaAs, structure sizes up to $100 \mu \mathrm{m}$ and in the case of $2 \mathrm{H}-\mathrm{GaN}$, structure sizes up to $40 \mu \mathrm{m}$ and photon energies approximately $10 E_{\mathrm{Urb}}$ below the spectrum peak energy $E_{\mathrm{m}}$ must be considered.

\subsection{Numerical simulation of a $p-i-n$ structure}

In order to check the presented predictions in the case of a real structure below full drift-diffusion, 1D numerical simulation results for $40 \mu \mathrm{m}$ basewidth GaAs and 2H-GaN $p-i-n$ structures are compared. The Shockley-Read-Hall lifetimes 12.8 and $30 \mathrm{~ns}$ are specified repectively for GaAs and $2 \mathrm{H}-\mathrm{GaN}$ in order to obtain (nonradiative) ambipolar diffusion length $5 \mu \mathrm{m}$ in the base layer. In $5 \mu \mathrm{m}$ thick emitter layers 10 times shorter SRH-lifetimes were assumed. The doping concentrations in $p$ - and $n$-emitters as well as in the base layer were taken equal to $10^{19} \mathrm{~cm}^{-3}$. The radiative recombination $R_{\mathrm{r}}$ and photon recycling generation $G_{\mathrm{pr}}$ were modelled as described above. All additional finer mechanisms like Auger-recombination, electron-hole scattering and band-gap narrowing were excluded. The electrical band-gaps 1.424 and $3.42 \mathrm{eV}$ were used for $\mathrm{GaAs}$ and $2 \mathrm{H}-\mathrm{GaN}$, respectively. Calculations were performed for the room temperature $300 \mathrm{~K}$. For comparison some results for the fixed local balance $G_{\mathrm{pr}} / R_{\mathrm{r}}=0.95$ are presented. This approach has been earlier proposed for practical GaAs power device calculations $\left.{ }^{31}\right]$.

The main results of simulations are presented in Figs. 7 and 8. 


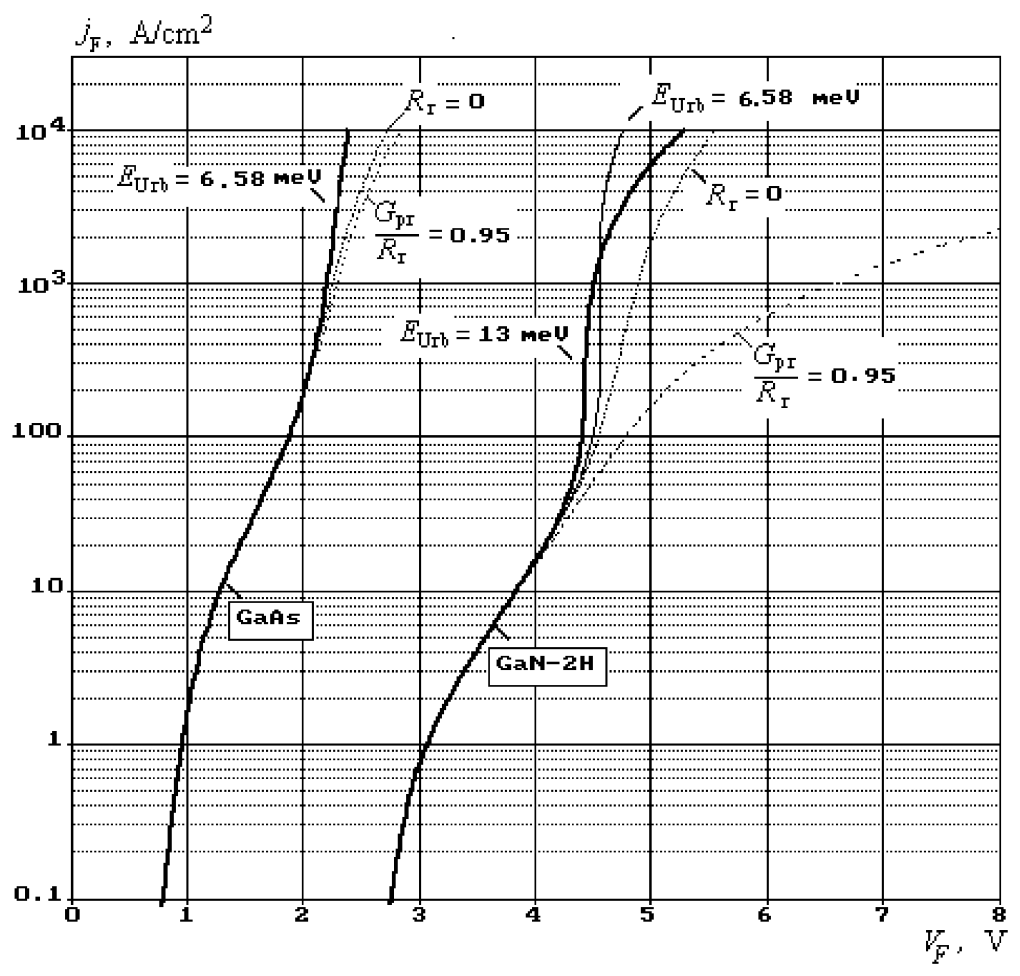

Fig. 7. Forward $I / V$ curves for GaAs and $2 \mathrm{H}-\mathrm{GaN} p-i-n$ structures calculated by $1 \mathrm{D}$ drift-diffusion device simulator DYNAMIT-1DT.

In general the obtained results confirm theoretical predictions. Both $I / V$ curves and internal carrier distributions confirm that in the case of $2 \mathrm{H}-\mathrm{GaN}$ the photon recycling effect is approximately one order of magnitude stronger than in the case of GaAs. Although the internal local ratios $G_{\mathrm{pr}} / R_{\mathrm{r}}$ in the vicinity of the carrier distribution minima are much greater in the case of GaAs (Fig. 8), the hole distributions in the base region of a GaAs structure are only moderately affected by the photon recycling even at high current density equal to $10^{4} \mathrm{~A} / \mathrm{cm}^{2}$. In contrast to that, in the $2 \mathrm{H}-\mathrm{GaN}$ structure the hole concentration distributions at high forward current densities are drastically changed by the photon recycling effect (Fig. 8). The S-shape $I / V$ curves are obtained for GaN in the case of both Urbach energies, $13 \mathrm{meV}$ (estimated $2 \mathrm{H}-\mathrm{GaN}$ value) and $6.58 \mathrm{meV}$ (standard GaAs value for comparison). By comparing curvatures of $p(x)$ at $10^{4} \mathrm{~A} / \mathrm{cm}^{2}$, it may be estimated that the photon recycling effect increases the effective diffusion length approximately $10 \%$ in the case of GaAs and several times in the case of $2 \mathrm{H}-\mathrm{GaN}$.

Simulation of the $2 \mathrm{H}-\mathrm{GaN}$ device demonstrates clearly that the simplified local balance $\left(G_{\mathrm{pr}} / R_{\mathrm{r}}=\right.$ const $)$ approach totally conflicts with full photon recycling model results. While the photon recycling lowers the carrier distribu- 

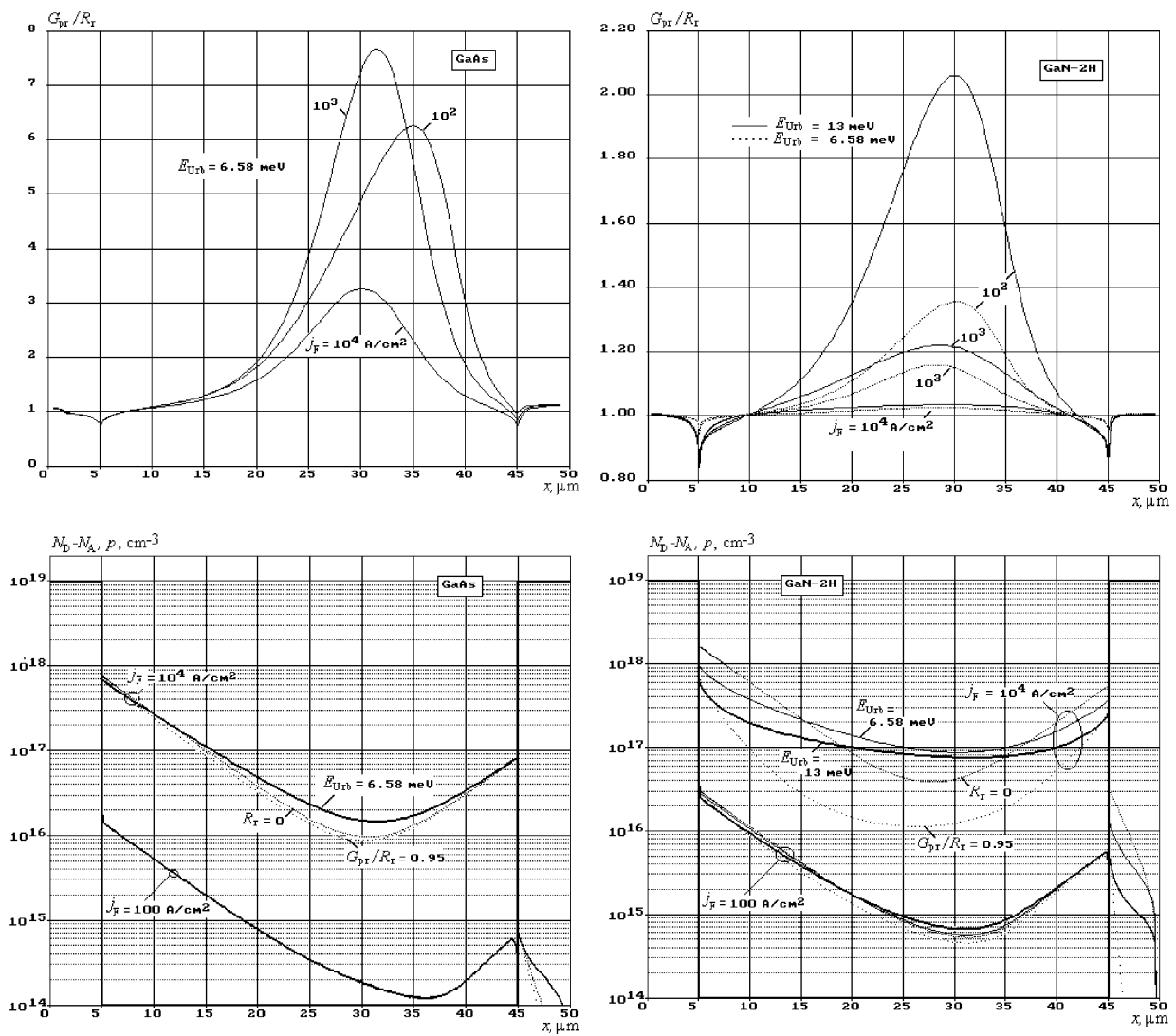

Fig. 8. Comparison of calculated hole concentration distributions and radiative generation/recombination ratio distributions $G_{\mathrm{pr}} / R_{\mathrm{r}}$ for GaAs and $2 \mathrm{H}-\mathrm{GaN} p-i-n$ structure at different forward current densities.

tions near the base region borders and fills central base area with additional charge carriers, this simplified approach fixes the strong unbalanced recombination channel with a very short effective lifetime due to the high radiative recombination coefficient $B$ of $\mathrm{GaN}$; that, in turn, essentially decreases charge carrier concentration in all the base area and drastically rises forward voltage drops.

It is worth to mention that some simulations were performed for the GaN structure with different basewidths. From those simulations it was found that basically the photon recycling has ambiguous effect when considering the appearance of the $S$-shaped $I / V$ curves. The filling of central base region with additional charge carriers decreases the voltage drops and facilitates the appearance of the $\mathrm{S}$-shape. The unbalanced strong radiative recombination near the base region borders decreases there effective lifetime and charge carrier concentration in the base area in general that increases the voltage drops and restricts appearance of the $\mathrm{S}$-shaped $I / V$ curves. 


\section{DISCUSSION AND CONCLUSION}

In this paper the strength of photon recycling effect was estimated for a new promising wide band-gap semiconductor $2 \mathrm{H}-\mathrm{GaN}$. Critical analysis showed that the necessary data for GaN are still very uncertain, especially in comparison with GaAs. The values of the key parameters like radiative recombination coefficient, given by different authors, vary for more than two orders of magnitude. However, we decided to use data which predict approximately one order of magnitude higher radiative recombination coefficient and absorption coefficients than in GaAs. Limited space of the present paper does not allow us to present here additional supporting calculations for this decision. Considering energy dependence of the absorption coefficient and radiation spectrum, we relied on the well-known Roosbroeck-Shockley formulation of the balance. Using this approach, the Urbach energy cannot exceed the value of thermal energy $k_{\mathrm{B}} T$. Following the paper by Muth et al. $\left[{ }^{20}\right]$, we decided to use Urbach energy $13 \mathrm{meV}$, which is approximately twice higher than established value for GaAs. With this discussion we want to emphasize that we have used rather small Urbach energy value for GaN as compared to $20-40 \mathrm{meV}$ used by some authors. For this reason it is possible that we have underestimated the contribution of the long-wave photons and thereby the effect of photon recycling in GaN. But even in the case of moderate Urbach energy our estimations show that in spite of high absorption coefficients the photon recycling effect in $\mathrm{GaN}$ should be approximately one order of magnitude stronger than in GaAs. For this comparison we have developed and offered here a methodology that is based on radiation recombination transfer function together with predictons for typical carrier distributions in the layers of the device. It should be mentioned that throughout this study we have used the assumption that the coefficient of internal photoeffect $\beta(E)$, i.e., probability that absorbed photon with energy $E$ creates an electron-hole pair is equal to 1 . This means actually nearly lossless photon recycling and in this respect we have studied the optimistic estimations. Unfortunately, very limited information may be found from literature about the behaviour of $\beta$. Limited space allowed us to present here only one example of numerical simulation of a $p-i-n$ structure. However, this example confirms the overall conclusion that in $\mathrm{GaN}$ the effect of photon recycling is probably much stronger than in GaAs.

\section{ACKNOWLEDGEMENT}

This work has been supported by the Estonian Science Foundation (grants Nos. 5180 and 5911).

\section{REFERENCES}

1. Dumke, W. P. Spontaneous radiative recombination in semiconductors. Phys. Rev., 1957, 105, 139-144. 
2. Stern, F. and Woodall, J. M. Photon recycling in semiconductor lasers. J. Appl. Phys., 1974, 45, 3904-3906.

3. Alferov, Zh. I., Andreev, V. M., Garbuzov, D. Z., Zhilyaev, Yu. V., Morozov, E. P., Portnoj, E. L., and Trofim, V. G. Investigation of the influence of the AlAs-GaAs heterostructure on the laser threshold current and the realization of continuous emission at the room temperature. Fiz. Tekhn. Polupr., 1970, 4, 1826-1829 (in Russian).

4. Alferov, Zh. I., Korol'kov, V. I., Nikitin, V. G., Stepanova, M. N., and Tret'yakov, D. N. Highspeed power diodes based on GaAs. Pis'ma Zhurn. Tekhn. Fiz., 1976, 2, 201-204 (in Russian).

5. Korol'kov, V. I., Nikitin, V. G., and Rakhimov, N. Thyristor based on $\mathrm{GaAs}_{-}-\mathrm{Al}_{\mathrm{x}} \mathrm{Ga}_{1-\mathrm{x}} \mathrm{As}$ heterostructures. Pis'ma Zhurn. Tekhn. Fiz., 1976, 2, 941-945 (in Russian).

6. Alferov, Zh. I., Bergmann, Ya. V., Korol'kov, V. I., Nikitin, V. N., Stepanova, M. N., Yakimenko, A. A., and Tret'yakov, D. N. Study on the direct branch of current-voltage characteristic of $p-n$ junctions based on highly doped GaAs. Fiz. Tekhn. Polupr., 1978, 12, 68-74 (in Russian).

7. Alferov, Zh. I., Korol'kov, V. I., Rakhimov, N., and Stepanova, M. N. Study on thyristors based on GaAs-AlGaAs heterostructures. Fiz. Tekhn. Polupr., 1978, 12, 75-81 (in Russian).

8. Korol'kov, V. I., Konicheva, I. M., Yuferev, V. S., and Yakovenko, A. A. Forward branch of the current-voltage characteristic of high-voltage diodes based on wide-band materials with direct band structure. Fiz. Tekhn. Polupr., 1978, 12, 1149-1153 (in Russian).

9. Alferov, Zh. I., Korol'kov, V. I., Konicheva, I. M., Yuferev, V. S., and Yakovenko, A. A. Effective control over the modulation of conduction of base region of gallium arsenide $p^{+}-n^{0}-n^{+}$-structures. Fiz. Tekhn. Polupr., 1979, 13, 271-280 (in Russian).

10. Durbin, S. M. and Gray, J. L. Numerical modeling of photon recycling in solar cells. IEEE Trans. Electron Devices, 1994, ED-41, 239-245.

11. Sieg, R. M. and Ringel, S. A. Reabsorption, band-gap narrowing, and the reconciliation of photoluminescence spectra with electrical measurements for epitaxial $n$-InP. J. Appl. Phys., 1996, 80, 448-458.

12. Parks Jr., J. W., Brennan, K. F., and Smith, A. W. Two-dimensional model of photon recycling in direct gap semiconductor devices. J. Appl. Phys., 1997, 82, 3493-3498.

13. Velmre, E. and Freidin, B. Numerical analysis of forward-biased diode structures based on direct-gap semiconductors. Electron. Lett., 1979, 15, 383-385.

14. Velmre, E., Freidin, B., and Udal, A. Numerical analysis of the on-state of diode structures based on direct-gap semiconductors. Phys. Scr., 1981, 24, 468-471.

15. Seidman, T. I. and Choo, S. C. Iterative scheme for computer simulation of semiconductor devices. Solid-State Electron., 1972, 15, 1229-1235.

16. Velmre, E., Udal, A., and Freidin, B. Investigation of efficiency of numerical algorithms for modeling of power semiconductor structures in the on-state. Elektronnoe Modelirovanie, 1981, 3, 85-88 (in Russian).

17. Varshni, Y. P. Band-to-band radiative recombination in groups IV, VI, and III-V semiconductors (I) and (II). Phys. Status Solidi, 1967, 19, 459-514 and 1968, 20, 9-36.

18. Im, J. S., Moritz, A., Steuber, F., Härle, V., Scholz, F., and Hangleiter, A. Radiative carrier lifetime, momentum matrix element, and hole effective mass in GaN. Appl. Phys. Lett., 1997, 70, 631-633.

19. Dmitriev, A. and Oruzheinikov, A. The rate of radiative recombination in the nitride semiconductors and alloys. J. Appl. Phys., 1999, 86, 3241-3246.

20. Muth, J. F., Lee, J. H., Shmagin, I. K., Kolbas, R. M., Casey Jr., H. C., Keller, B. P., Mishra, U. K., and DenBaars, S. P. Absorption coefficient, energy gap, exciton binding energy, and recombination lifetime of GaN obtained from transmission measuremen. Appl. Phys. Lett., 1997, 71, 2572 2574.

21. van Roosbroeck, W. and Shockley, W. Photon-radiative recombination of electrons and holes in germanium. Phys. Rev., 1954, 94, 1558-1560. 
22. Knobloch, K., Perlin, P., Krueger, J., Weber, E. R., and Kisielowski, C. Effect of internal absorption on cathodoluminescence from GaN. Internet J. Nitride Semicond. Res., 1998, 3, 4 (http://nsr.mij.mrs.org/3/4/).

23. Casey Jr., H. C., Sell, D. D., and Wecht, K. W. Concentration dependence of the absorption coefficient for $n$ - and $p$-type GaAs between 1.3 and 1.6 eV. J. Appl. Phys., 1975, 46, 250257.

24. Velmre, E. Approximate calculation of carrier transfer factor under band-to-band recombination radiation reabsorption conditions. Proc. Estonian Acad. Sci. Phys. Math., 1984, 33, 87-94 (in Russian).

25. Hakki, B. W. Theory of luminescent efficiency of ternary semiconductors. J. Appl. Phys., 1971, 42, 4981-4995.

26. Kuriyama, T., Kamiya, T., and Yanai, H. Effect of photon recycling on diffusion length and internal quantum efficiency in $\mathrm{Al}_{\mathrm{x}} \mathrm{Ga}_{1-\mathrm{x}} \mathrm{As}-\mathrm{GaAs}$ heterostructures. Jpn. J. Appl. Phys., 1977, 16, 465-477.

27. Mettler, K. Effect of reabsorbed recombination radiation on photoluminescence and photoconductivity in semi-infinite direct-gap semiconductor. Phys. Status Solidi (a), 1978, 49, $163-168$.

28. Urbach, F. The long-wavelength edge of photographic sensitivity and of the electronic absorption of solids. Phys. Rev., 1953, 92, 1324.

29. Sell, D. D., Casey Jr., H. C., and Wecht, K. W. Concentration dependence of the refractive index for $n$ - and p-type GaAs between 1.2 and $1.8 \mathrm{eV}$. J. Appl. Phys., 1974, 45, 2650-2657.

30. Ejder, E. Refractive index of GaN. Phys. Status Solidi (a), 1971, 6, 445-448.

31. Alferov, Zh. I., Ashkinazi, G. A., Korol'kov, V. I., Pad'yus, A. L., Timofeev, V. N., Chelnokov, V. E., and Shumilin, V. N. Modulation of the conduction of a lightly doped $N$-region of $P^{+}-N-N^{+}$-structures based on straight-band semiconductors. Fiz. Tekhn. Polupr., 1978, 12, 1336-1341 (in Russian).

\section{Footonite tagasineeldumise võrdlus GaAs- ja GaN-struktuurides}

\section{Enn Velmre ja Andres Udal}

Varasemate, peamiselt GaAs-iga seotud uuringute edasiarendusena on käsitletud võrdlevalt tsoonidevahelise rekombinatsioonkiirguse tagasineeldumist GaAs- ja GaN-struktuurides. Kirjanduse andmetel on GaN-i rekombinatsioonkiirguse ja optilise kiirguse neeldumise tegurid umbes kümme korda suuremad kui GaAs-il, kusjuures pikalainelise kiirguse neeldumisspektrit iseloomustava nn Urbachi energia kohta on andmed vastukäivad. Materjalide võrdlust hõlbustavat rekombinatsioonkiirguse ülekandefunktsiooni $F(u)$ uuriti erinevate neeldumisteguri väärtuste ja rekombinatsioonkiirguse pikalainelise spektriosa laiuste korral. Tulemused näitavad, et tugevale neeldumisele vaatamata on footonite tagasineeldumise efektiivsus $\mathrm{GaN}$-is umbes suurusjärgu võrra kõrgem kui GaAs-is. Suurtel voolutihedustel võib see põhjustada $p-i-n$-struktuuri pinge-voolukarakteristiku S-kujulise kõverdumise. 